\title{
An Image Watermarking Based on the pdf Modeling and Quantization Effects in the Wavelet Domain
}

\author{
Irena Orović, Milica Orlandić and Srdjan Stanković \\ Faculty of Electrical Engineering, University of Montene gro \\ +38267516795 \\ +38220242667 \\ (irenao, milica, srdjan)@ac.me
}

\begin{abstract}
An image watermarking technique based on the concept of JPEG2000 algorithm is proposed. Biorthogonal wavelet $9 / 7$ transform is used to provide a set of coefficients suitable for watermark embedding. The statistical properties of different subbands are analyzed in order to choose the number of decomposition levels and position of subbands, which will assure the best compromise between the watermark transparency and robustness. The JPEG2000 quantization is applied to avoid insignificant wavelet coefficients, while the remaining ones are used for watermarking. The optimal and blind watermark detection is based on the nonlinear score function and appropriate model of coefficients distribution. The performance of the proposed procedure is tested on examples with various images, showing robustness under different attacks, while maintaining high image quality.
\end{abstract}

Index terms - image watermarking, wavelet transform, JPEG2000 quantization, optimal detection

\section{INTRODUCTION}

The intensive development of digital technology brings a demanding task of digital data protection. Among different protection techniques, digital watermarking appears as an effective solution. Various watermarking techniques have been developed depending on the data type [1],[2]. If the watermarking procedure is used for ownership protection, it should satisfy two important requirements: imperceptibility and robustness.

Here we focus on watermarking procedure for image protection. Watermarking techniques mainly consist of the following steps: watermark design, embedding and watermark detection. Watermark is usually created as a pseudorandom sequence. In order to efficiently protect the host data, it should be robust to non-malicious and malicious signal processing techniques called 
attacks. The watermark that can be detected even after attack is called robust. On the other hand, to be imperceptible, the watermark strength should be weak. Thus, the efficiency of the watermarking procedure is based on a good compromise between the imperceptibility and robustness. Image watermarking can be performed within the spatial domain or some of the transform domains [2]-[6]. The discrete cosine transform (DCT) coefficients are frequently used for image watermarking [6]-[8], particularly the middle frequency DCT coefficients from $8 \times 8$ blocks. Based on different statistical models of coefficients, various optimal detector forms have been proposed, such as detector based on the generalized Gaussian function (GGF) [6], nonlinear detectors based on the Cauchy model [8],[9], etc. An approach to image watermarking in the presence of JPEG quantization is considered in [10]. The influence of JPEG quantization effects on watermarked DCT coefficients and watermark itself has been analyzed. The proposed procedure uses an improved form of optimal detector providing robustness under various quantization degrees and other commonly used attacks. With the development of JPEG2000, the discrete wavelet transform (DWT) based watermarking methods become more interesting [11][16]. The optimal parameter selection and their impact on the efficiency of DWT based image watermarking has been studied in [11],[12]. The DWT based techniques provides good spacefrequency localization and superior human visual system (HVS) modeling [13]. For instance, following the HVS directives, the algorithm proposed in [14] embeds the watermark into highfrequency subbands of the three level decomposition DWT coefficients. Both the watermark and watermarked coefficients are chosen depending on the statistic function values of the image. The detection is performed using the correlation between watermarked coefficients and watermark according to the Neyman-Pearson criterion. In [15], a watermarking performance is tested in both low and high frequencies. It has been shown that embedding in low frequencies increases the robustness with respect to one group of attacks such as filtering, lossy compression, geometric distortions, but makes the procedure sensitive to modifications of the image histogram. On the other side, watermark embedded in the high frequencies can be robust with respect to noise and nonlinear deformations of the gray scale. Hence, the performances of DWT-based procedures depend on the coefficients and subband level chosen for watermark embedding.

By embedding the watermark in the same domain as the compression scheme, it is possible to anticipate which transform coefficients will be discarded by compression [17], [18], and consequently to provide better watermark robustness. Particularly, let us discuss some of the 
algorithms based on the JPEG2000 compression. The JPEG2000 quantization is usually applied to the coefficients obtained at the output of 9/7 taps filter bank, and then a suitable set of nondiscarded coefficients is chosen for watermarking. On the one hand, the watermark can be detected or extracted in the presence of original image [17],[19],[20], which is usually available only to the owner (non-blind detection). On the other hand, blind watermark detection can be done as in [21], where a watermark bit is embedded by shifting the selected coefficients to a quantization index (depending on the watermark bit). The watermark bit is detected using the value of quantization step: even (odd) step corresponds to watermark bit "0" ("1"). However, in this case, to provide watermark robustness a stronger quantization should be applied during watermark embedding process, which results in lower PSNR (around $30 \mathrm{~dB}$ ). Hence, the existing JPEG2000 based watermarking methods should be improved in the sense to provide blind and robust watermark detection, while maintaining high quality of the watermarked image (with PSNR above $45 \mathrm{~dB}$ ).

A robust watermarking algorithm, that is compliant and compatible with JPEG2000, is proposed in this paper. It represents an extension of the watermarking procedure done in [10] that uses DCT domain and examines the influence of JPEG quantization. The biorthogonal $(9,7)$ floating point wavelets, as in the JPEG2000, are used for watermarking. The advantage of biorthogonal over other wavelet families is found in the symmetry of the filter coefficients providing linear phase of transfer function. The lifting scheme additionally simplifies the realization of wavelet transform [22],[23], which is a reason for using this scheme. A suitable subband level for watermark embedding is chosen to provide the best robustness-imperceptibility compromise. Then, the coefficients selection method is examined, in order to provide robustness in the presence of various attacks. The watermark detection is based on a specific form of coefficients pdf that is preserved even after attack. Consequently, an optimal detector form is used to provide reliable detection results. At the same time, the procedure provides high values of quality metrics for watermarked image, ensuring the watermark imperceptibility. The efficiency of the proposed scheme is tested on various images and under different attacks. The proposed procedure improves the performance of its counterpart in the JPEG based DCT domain, as well as the performance of some standard DWT based approaches.

The paper is organized as follows. The procedure for coefficients selection and watermark embedding is described in Section 2. The watermark detection is considered in Section 3. The 
experimental results, demonstrating the efficiency of the proposed approach, are given in Section 4. The concluding remarks are given in Section 5.

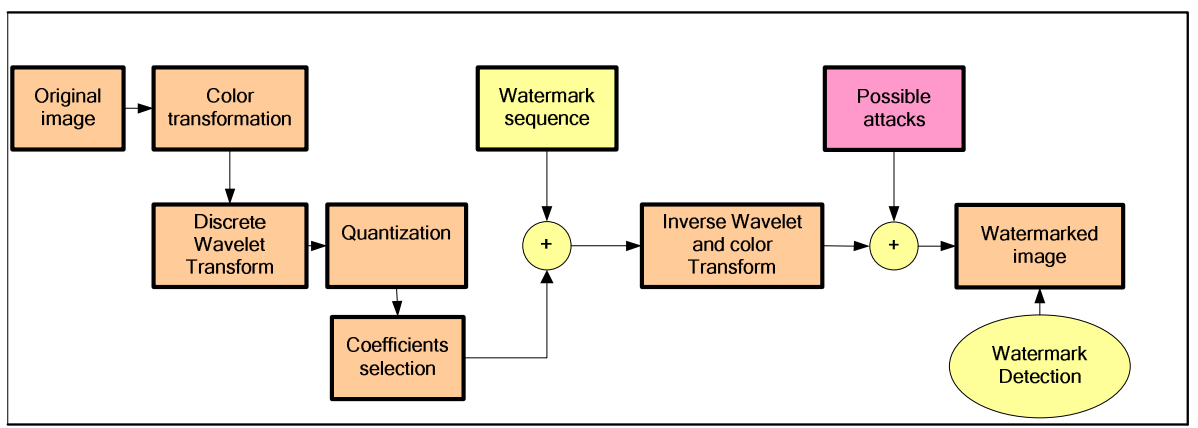

Fig 1. Block diagram of the watermarking procedure

\section{WATERMARKING PROCEDURE IN DWT DOMAIN}

The block scheme of the proposed watermarking procedure is presented in Fig.1. It can be summarized through the following steps: 1) The color transform and DWT are applied to the original image; 2) JPEG2000 quantization is applied to DWT coefficients belonging to appropriate decomposition subbands; 3) A suitable coefficients selection is defined on the basis of quantization parameters; 4) Watermark is created as a pseudo-random sequence and embedded in the selected coefficients; 5) The optimal watermark detection is based on statistical modelling of coefficients pdf. The details of implementation and the criteria for parameters selection will be described in the sequel. Note that this approach aims to provide a watermarking procedure with highly controlled performance in terms of its robustness and imperceptibility. This is achieved through the appropriate selection of decomposition levels, quantization parameters and the choice of subband coefficients that will preserve pdf shape in the presence of attacks.

A color image is transformed to $\mathrm{YCbCr}$ domain. It has been shown in [11] that the $\mathrm{YCrCb}$ system can be acceptable candidate for most of the wavelet based watermarking algorithms. Also, it provides significant tolerance against JPEG compression and noise addition, especially when the watermark is hidden in the Y channel [12]. Thus, in the proposed procedure, we will focus on the luminance component. Furthermore, the discrete wavelet transform is performed by using the biorthogonal discrete $(9,7)$ floating point wavelets in order to be compliant with JPEG2000. The other important reason is that the biorthogonal wavelet transform allows implementation by using a lifting scheme, which significantly simplifies the realization [22],[23]. Otherwise, the DWT is 
implemented as a convolution or the finite impulse response filter bank structures that require large number of arithmetic computations and large storage. Hence, the traditional implementations could be unpractical for high speed or low power image/video processing applications [24].

In the wavelet decomposition, the low frequency subbands carry a large amount of image energy and their manipulation could produce perceptual quality degradation. On the other hand, high frequency subbands suffer from the processing noise. Hence, the coefficients from the middle frequency subbands are expected to be a suitable choice.

In order to select a set of wavelet coefficients appropriate for watermarking, we employ the embedded scalar quantization used in JPEG2000. The scalar quantization is performed only on the coefficients of luminance component denoted by $I(x, y)$. The values of the coefficients after the quantization are obtained as follows [25]:

$$
I_{q}(x, y)=K^{p}(x, y) q
$$

where $q$ represents the quantization step, while the quantization index $K^{p}$ is the output of the uniform quantizer: $K^{p}=Q(I(x, y))$, and for a given quantization step it is calculated according to:

$$
K^{p}(x, y)=\operatorname{sgn}(I(x, y))\left\lfloor\frac{|I(x, y)|}{q}\right\rfloor .
$$

Hence, for a given wavelet coefficient $I(x, y)$, the quantizer produces a signed integer $K^{p}$. The quantization index $K^{p}$ indicates the interval in which $I(x, y)$ lies.

The notation $\lfloor x\rfloor$ refers to the greatest integer less than or equal to $x$. Each subband has different quantization step, which decreases by factor 2 as subband level increases. The quantization step can be defined as:

$$
q=\frac{R_{\max }-R_{\min }}{2^{p+i}},
$$

where $\left[R_{\min }, R_{\max }\right]$ represents the dynamic range of wavelet coefficients, while $i$ indicates the subband level. Parameter $p$ controls the image quality. Namely, higher values of $p$ provide better image quality, but lower compression ability. Thus, the smallest $p$, that does not produce perceptual image degradation, should be used. Consequently, the quantization effects are examined for different values of parameter $p$ and for different numbers of decomposition levels (4, 5 and 6 levels). The image quality after the quantization has been tested by using the following quality metrics: peak signal to noise ratio (PSNR), visual signal to noise ratio (VSNR) [26], 
composite-peak-signal-to-noise-ratio (CPSNR) [27], and subjective evaluation by human observers (with binary decision "passed" or "failed"). The results are presented in Table 1. Note that for $p>8$, the values of quality metrics indicate good image quality for all tested decomposition levels. Thus, $p=9$ is used in the implementation of the procedure.

Table 1 . The influence of parameter $p$ on image quality

\begin{tabular}{|c|c|c|c|}
\hline & 4 le & 5 le & $6 \mathrm{le}$ \\
\hline$p=6$ & $\begin{array}{l}\mathrm{PSNR}=34 \\
\mathrm{VSNR}=36 \\
\mathrm{CPSNR}=34.68 \\
\text { subjective=Failed }\end{array}$ & $\begin{array}{l}\mathrm{PSNR}=30.68 \\
\mathrm{VSNR}=30.95 \\
\mathrm{CPSNR}=31.9 \\
\text { subjective=Failed }\end{array}$ & $\begin{array}{l}\text { PSNR=29.34 } \\
\text { VSNR=25.96 } \\
\text { CPSNR=29.63 } \\
\text { subjective=Failed }\end{array}$ \\
\hline$p=7$ & $\begin{array}{l}\mathrm{PSNR}=38 \\
\mathrm{VSNR}=39.7 \\
\text { CPSNR }=38.35 \\
\text { subjective=Passed }\end{array}$ & $\begin{array}{l}\text { PSNR=34.68 } \\
\text { VSNR=36.7 } \\
\text { CPSNR=35 } \\
\text { subjective=Passed }\end{array}$ & $\begin{array}{l}32 \\
=31.7 \\
=32.3 \\
\text { ve=Failed }\end{array}$ \\
\hline$p=8$ & \begin{tabular}{|l|}
$\mathrm{PSNR}=42.43$ \\
$\mathrm{VSNR}=40.8$ \\
CPSNR $=42.75$ \\
subjective $=$ Passed \\
\end{tabular} & \begin{tabular}{|l|}
$\mathrm{PSNR}=38.4$ \\
$\mathrm{VSNR}=40$ \\
CPSNR $=38.7$ \\
subjective=Passed \\
\end{tabular} & \begin{tabular}{|l|}
$\mathrm{PSNR}=35.17$ \\
$\mathrm{VSNR}=37.5$ \\
CPSNR $=35.4$ \\
subjective=Passed \\
\end{tabular} \\
\hline$p=9$ & $\begin{array}{l}\mathrm{PSNR}=47.4 \\
\mathrm{VSNR}=40.5 \\
\mathrm{CPSNR}=47.75 \\
\text { subjective }=\text { Passed }\end{array}$ & $\begin{array}{l}\mathrm{PSNR}=42.9 \\
\mathrm{VSNR}=40.8 \\
\mathrm{CPSNR}=43 \\
\text { subjective }=\text { Passed }\end{array}$ & $\begin{array}{l}\mathrm{PSNR}=39 \\
\mathrm{VSNR}=40.8 \\
\mathrm{CPSNR}=40 \\
\text { subjective }=\text { Passed }\end{array}$ \\
\hline$p=10$ & $\begin{array}{l}\mathrm{PSNR}=53 \\
\mathrm{VSNR}=42.7 \\
\mathrm{CPSNR}=53.3 \\
\text { subjective= Passed }\end{array}$ & $\begin{array}{l}\mathrm{PSNR}=47.7 \\
\mathrm{VSNR}=41 \\
\mathrm{CPSNR}=48.3 \\
\text { subjective= Passed }\end{array}$ & $\begin{array}{l}\mathrm{PSNR}=43.6 \\
\mathrm{VSNR}=41 \\
\mathrm{CPSNR}=44 \\
\text { subjective= Passed }\end{array}$ \\
\hline
\end{tabular}

According to the JPEG2000, the inverse quantization is done by:

$$
\tilde{I}=\left\{\begin{array}{ll}
0, & K^{p}=0 \\
\operatorname{sgn}\left(K^{p}\right)\left(\left|K^{p}\right|+\delta\right) q, & K^{p} \neq 0
\end{array} .\right.
$$

The bias parameter $\delta$ can be chosen to achieve the best subjective or objective quality at reconstruction. It takes values from the range $0 \leq \delta<1$. Although the value of $\delta$ is not normatively specified in the standard, usually $\delta=0.5$ is used [25], [28].

The coefficients whose values are in the range $[-q, q]$ will be zero after scalar quantization. These coefficients belong to the so called dead zone and should be avoided in the watermarking procedure. The rest of the coefficients can be selected for watermarking and can be defined as:

$$
\left|I_{q}(x, y)\right|>C \cdot q,
$$


where $C \geq 1$ will be called the gap parameter and $q$ is the quantization step. The corresponding pdf of coefficients is shown in Fig.2. Note that the specific pdf form is obtained, which is not of the continual form like the commonly used Gaussian or Laplacian pdf. It consists of two parts (Fig 2):

- $\quad$ the central part of pdf function (thick line),

- $\quad$ the decaying tails (dashed line) that correspond to the tails of Gaussian function.

Hence, the coefficients' pdf can be modeled as [10]:

$$
\xi\left(I_{w}\right)=\left(\frac{\left(\frac{I_{q}}{a}\right)^{2 n}}{\left(\frac{I_{q}}{a}\right)^{2 n}+1}\right)\left(e^{-\left|\frac{I_{q}}{a}\right|^{2}}\right) .
$$

Parameter $a$ defines the position of the pdf maximum, and it is calculated as: $a=\arg \max (H)$, where $\mathrm{H}$ is the histogram of coefficients. Parameter $n$ controls the decay of function $f\left(I_{q}\right)=\left(I_{q} / a\right)^{2 n} /\left(\left(I_{q} / a\right)^{2 n}+1\right)$ between the histogram maximum and origin. For most of the tested images $n$ takes either value 3 or value 4 . Note that $f\left(I_{q}\right) \rightarrow 1$ for $\left|I_{q}\right|>|a|$ and $n \gg 1$, and the pdf approaches to Gaussian form in this region.

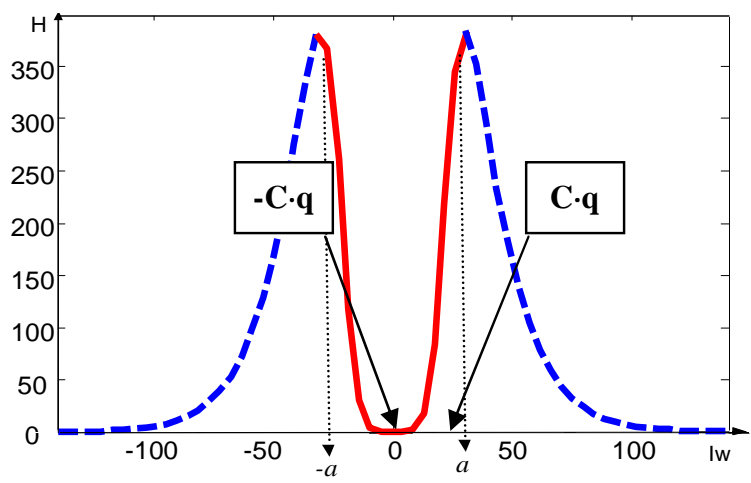

Fig 2. The pdf of selected coefficients

In order to provide optimal detection based on the coefficients pdf, it is important to retain the shape of pdf after attack. The detector performance will depend on the value of gap parameter $C$. Namely, for small $C$ the gap may disappear under attack, which will decrease the detector performance. As an illustration, let us consider two examples where the pdf is modified after 
adding a Gaussian noise, Fig 3. In the first case, the original pdf is obtained for $C=10$ (Fig 3.a), and it is changed under attack such that the dead zone has disappeared (Fig 3.b). In the second example, a larger $C(C=40)$ is considered (Fig 3.c). Note that the pdf shape is slightly changed under attack, but the gap is still preserved (Fig 3.d).

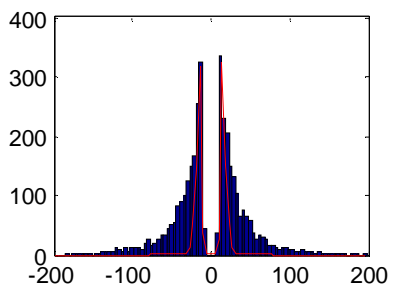

(a)

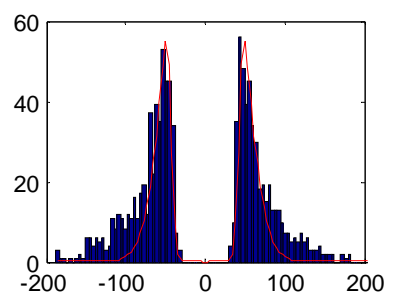

(c)

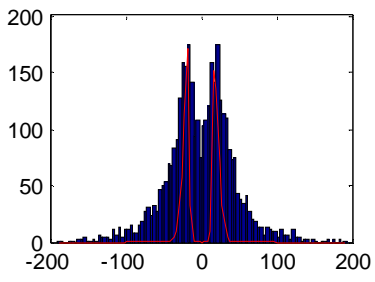

(b)

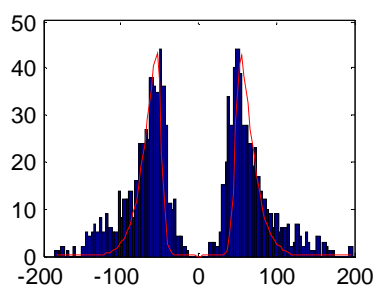

(d)

Fig 3. $C=10$ : a) pdf before attack, b) pdf after attack; $C=40$ : c) pdf before attack, d) pdf after attack

Another important issue is the choice of subband level from which the coefficients should be selected. Having in mind that watermark in low frequency subbands could easily degrade imperceptibility, the middle and high frequency subbands are examined. Based on the set of experiments, it has been shown that the best imperceptibility-robustness compromise is achieved by using the coefficients from the third (III) subbands of the 5 level decomposition scheme (Fig. 4). A detailed description of results is given in the following section.

\begin{tabular}{|c|c|c|}
\hline 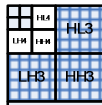 & $\mathrm{HL} 2$ & HL1 \\
\hline LH2 & $\mathrm{HH} 2$ & \\
\hline \multicolumn{2}{|c|}{ LH1 } & HH1 \\
\hline
\end{tabular}

Fig 4. Subbands of interest 
The multiplicative embedding procedure is performed as follows:

$$
I_{w}=I_{q}+\alpha\left|I_{q}\right| w
$$

where $I_{w}$ is the watermarked image, while $w$ is the watermark created as a pseudo-noise sequence (randomly generated real numbers having a normal distribution with zero mean and unity variance). The watermark strength is controlled by the scaling factor $\alpha$.

\section{WATERMARK DETECTION}

The Locally Optimal (LO) Detector form, which is well suited to watermark detection, can be obtained according to [29]:

$$
D=\sum_{i=1}^{K} w_{i} g_{l o}\left(I_{w_{i}}\right)
$$

where $K$ is the length of watermark sequence $w$. The non-linear score function $g_{l o}$ is defined as:

$$
g_{l o}\left(I_{w}\right)=-\frac{\xi^{\prime}\left(I_{w}\right)}{\xi\left(I_{w}\right)},
$$

where $\xi\left(I_{w}\right)$ and $\xi^{\prime}\left(I_{w}\right)$ represent the pdf of watermarked coefficients and its first derivative, respectively. By using the non-continuous pdf form defined by (6), the optimal detector is defined as [7]:

$$
D_{o p t}=\sum_{i=1}^{K} w_{i}\left(I_{w_{i}}-\frac{n \alpha^{2}}{I_{w_{i}}\left(1+\left(\frac{I_{w_{i}}}{\alpha}\right)^{2 n}\right)}\right) .
$$

As a measure of detection quality and reliability, we use the detectability or discriminability index from the signal detection theory [30]. It has been introduced to discriminate between signal and non-signal by measuring the separation and the spread of the noise-alone and signal-plus-noise curves: $\mathrm{M}=$ separation/spread. In watermarking, we have to discriminate between hits (watermarks) and false alarms (wrong trials). Hence, the measure of the detection quality is defined as: 


$$
M=\frac{\bar{D}_{\text {wrong }}-\bar{D}_{w}}{\sqrt{\sigma_{\text {wrong }}^{2}+\sigma_{w}^{2}}},
$$

where $\bar{D}$ and $\sigma$ are the mean value and standard deviation of the detector responses, while $w$ and wrong indicate watermarks (keys) and wrong trials, respectively. Here, wrong trial can be any arbitrary sequence, created in the same way as watermark, but not embedded in the host data. Higher values of measure $M$ provide more reliable detection. For example $M>5$ provides $\mathrm{P}_{\text {err }}>10^{-5}$. The performance of the optimal detector will be compared with the commonly used standard correlation detector:

$$
D=\sum_{i=1}^{K} w_{i} g_{l o}\left(I_{w_{i}}\right)=\sum_{i=1}^{K} w_{i} I_{w_{i}}
$$

Note that the correlation detector is defined by using the non-linear score function given by (9), where it is assumed that the watermarked coefficients has Gaussian pdf, i.e., $\xi\left(I_{w}\right)$ is Gaussian function. The standard correlation detector has been widely used in the literature due to the simplicity of realization (even in on-line applications).

Additionally, we will provide an example where the proposed approach is compared with detectors based on the Generalized Gaussian and Cauchy pdf.

\section{EXPERIMENTAL RESULTS}

\subsection{Example 1}

In order to prove the efficiency of the proposed system, a set of images is considered in the experiments. This set includes commonly used test images such as Lena, Barbara, Baboon, Airplane, Peppers, Boats, Goldhill, house, etc. The original and watermarked images Lena, Baboon and Peppers are presented in Fig. 5. The achieved PSNR is high and approximately around $47 \mathrm{~dB}$ for all tested images $(C P S N R \approx 48 \mathrm{~dB}, V S N R \approx 38 \mathrm{~dB})$. The quantization step is calculated by using (3), where parameter $p$ is set on value 8 . The III subband of the five level decomposition scheme is used for watermarking. Namely, it is shown experimentally that the watermark embedding in the III subband produces the best results regarding the watermark detection performance. This is examined within the Example 2. 
The watermark detection is tested for 100 watermarks by using the optimal detector form. For each watermark, 100 wrong trials are generated. The measures of detection quality $(M)$ are calculated according to (7).

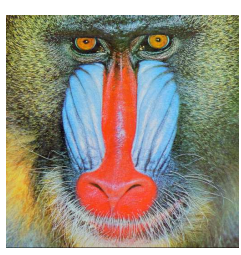

(a)

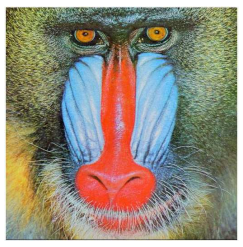

(d)

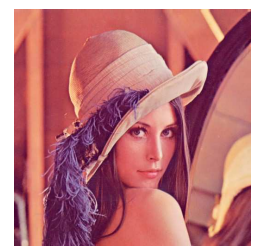

(b)

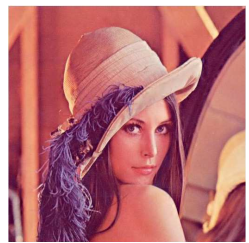

(e)

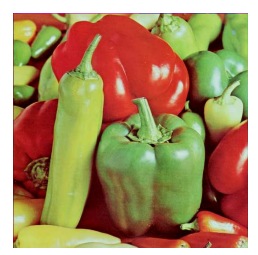

(c)

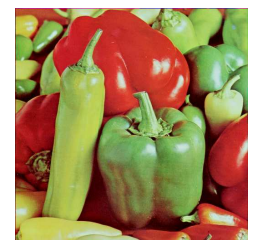

(f)

Fig 5. Original images: a) Mandrill, b) Lena, c) Peppers,

Watermarked images: d) Mandrill, e) Lena, f) Peppers

The image quality and watermark detectability are tested for different values of embedding strength $\alpha$ (e.g. $\alpha=0.05, \alpha=0.1, \alpha=0.2$ ). For each value of $\alpha$, the parameters PSNR, CPSNR and measure of detection quality $M$ are calculated. Also, different values of gap parameter $\mathrm{C}$ have been used. The results are given in Table 2 . It has been shown that $\alpha=0.1$ provides high values of PSNR and CPSNR, while providing satisfactory detection (measured by $M$ ). Thus, for a set of tested images, $\alpha=0.1$ provides the finest compromise between the imperceptibility and watermark detectability.

Table 2. PSNR, CPSNR and detection measure $M$ for different values of $\alpha$

\begin{tabular}{|c|c|c|c|c|}
\hline & $\mathbf{C = 2 0}$ & $\mathbf{C}=\mathbf{4 0}$ & $\mathbf{C}=\mathbf{6 0}$ & \\
\hline \multirow{3}{*}{$\boldsymbol{\alpha}=\mathbf{0 . 0 5}$} & 52.7879 & 53.4643 & 54.1732 & PSNR \\
& 53.4483 & 54.1266 & 54.8314 & CPSNR \\
\cline { 2 - 5 } & $\mathbf{5 . 9 9 7 4}$ & $\mathbf{4 . 9 2 0 0}$ & $\mathbf{3 . 9 3 4 7}$ & M \\
\hline \multirow{3}{*}{$=\mathbf{0 . 1}$} & 46.7458 & 47.1782 & 48.1437 & PSNR \\
& 47.4067 & 47.8378 & 48.8057 & CPSNR \\
\cline { 2 - 5 } & $\mathbf{1 0 . 3 0 4 1}$ & $\mathbf{9 . 2 6 6 4}$ & $\mathbf{6 . 1 7 8 4}$ & M \\
\hline \multirow{3}{*}{$\boldsymbol{\alpha}=\mathbf{0 . 2}$} & 40.6100 & 40.6458 & 42.0983 & PSNR \\
& 41.2620 & 41.2960 & 42.7560 & CPSNR \\
\cline { 2 - 6 } & $\mathbf{1 2 . 3 8 8 6}$ & $\mathbf{1 3 . 1 4 6 5}$ & $\mathbf{7 . 8 4 1 4}$ & M \\
\hline
\end{tabular}




\subsubsection{Robustness}

In the sequel, the robustness of the watermarking procedure is tested in the presence of different attacks, such as impulse noise with the variance 0.003 , Gaussian noise of variance 0.01 , median filtering, JPEG and JPEG2000 compressions with various compression factors.

Firstly, the performance of the proposed watermarking procedure is analyzed for different values of gap parameter $C$ in order to choose an optimal value $C_{\text {opt }}$ for all considered attacks. The detection measure in terms of the gap parameter $C$ is illustrated in Fig 6 for a sample test image Lena. Similar results are obtained for other test images. The results obtained by using the optimal detector are compared with the results of the standard correlation detector. For instance, in the case of Gaussian noise, low values of $C$ cause low performance of optimal detector. Note that for $C=40$, the measure of optimal detection under Gaussian noise takes its maximum, and provides high values of $M$ in the presence of other attacks, as well. Hence, an optimal value of $C$ that provides robustness for all considered attacks (even for the Gaussian noise) is $C_{\text {opt }}=40$.

Furthermore, for a set of tested images, the maximal, minimal and mean values of measure $M$ are calculated and presented in Table 3 for optimal detector and standard correlator. One can observe, from Table 3, that the optimal detector provides high values of $M$ even under attacks and significantly outperforms the standard correlation detector.

It is interesting to mention that the JPEG compression does not affect significantly the pdf of watermarked coefficients and thus, it does not degrade watermark detection even for low quality factor (e.g. QF=30\%). Moreover, the watermark sequence does not require any additional conditions to provide its detectability as it was the case in [10]. Also, the responses of optimal detector in the presence of attacks are improved.

Additionally, we have tested the robustness to the geometrical attacks: rotation, scaling and cropping. The results are presented in Table 4. The optimal detector provides high detection measures for cropping ( 3 row and 3 cols), image scaling to $90 \%$ and resize to the original dimensions, and rotation for 1 degree. 

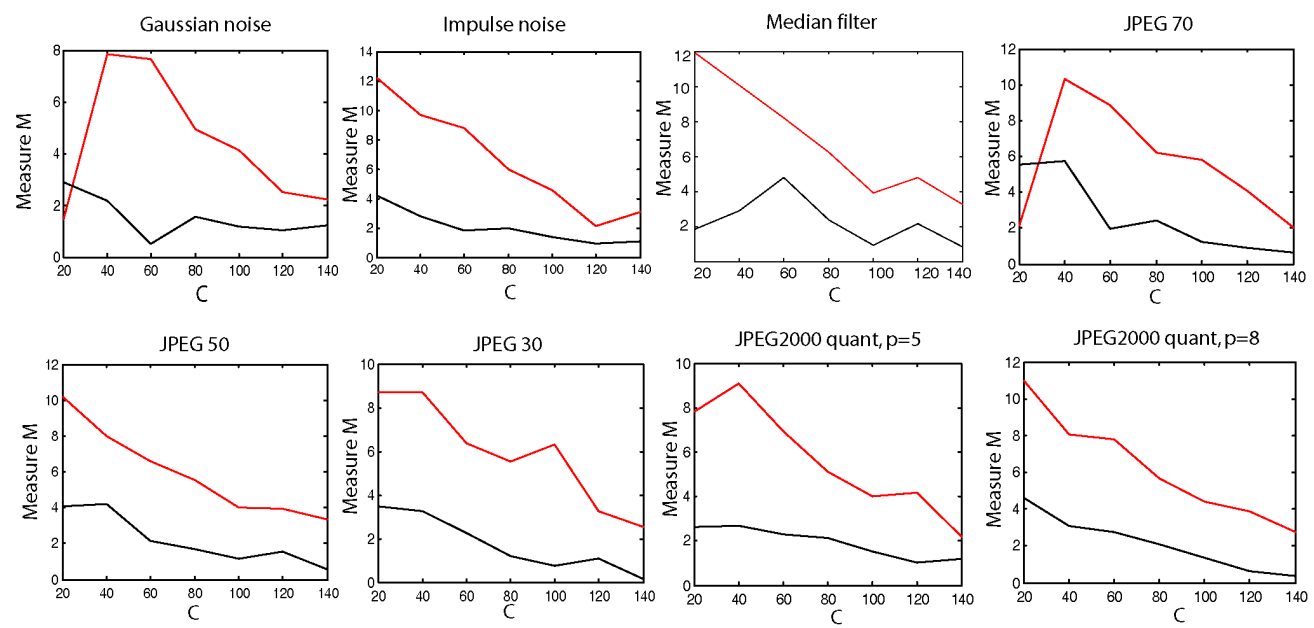

Fig 6. The detection measure $\mathrm{M}$ in terms of the gap parameter $\mathrm{C}$

Table 3 Maximal, minimal and mean detection measures $M$ for a set of tested images under commonly present attacks

\begin{tabular}{|c|c|c|}
\hline $\begin{array}{c}\text { Test } \\
\text { Statistics }\end{array}$ & $\begin{array}{l}\text { Standard } \\
\text { correlator }\end{array}$ & $\begin{array}{l}\text { Optimal } \\
\text { detector }\end{array}$ \\
\hline \multirow{3}{*}{ No attack } & $\mathrm{M}_{\min }=4.19$ & $\mathrm{M}_{\min }=10.5$ \\
\hline & $\mathrm{M}_{\text {mean }}=4.9$ & $\mathrm{M}_{\text {mean }}=13.5$ \\
\hline & $\mathrm{M}_{\max }=5.28$ & $\mathrm{M}_{\max }=18.5$ \\
\hline \multirow{3}{*}{ Gaussian noise } & $\mathrm{M}_{\min }=3.33$ & $\mathrm{M}_{\min }=6.22$ \\
\hline & $\mathrm{M}_{\text {mean }}=4.2$ & $\mathrm{M}_{\text {mean }}=7.5$ \\
\hline & $\mathrm{M}_{\max }=5.6$ & $\mathrm{M}_{\max }=8.43$ \\
\hline \multirow{3}{*}{ Impulse noise } & $\mathrm{M}_{\min }=3.8$ & $\mathrm{M}_{\min }=9.67$ \\
\hline & $\mathrm{M}_{\text {mean }}=4.6$ & $\mathrm{M}_{\text {mean }}=12$ \\
\hline & $\mathrm{M}_{\max }=7.04$ & $\mathrm{M}_{\max }=17.20$ \\
\hline \multirow{3}{*}{ Median filter } & $\mathrm{M}_{\min }=3.56$ & $\mathrm{M}_{\min }=10.2$ \\
\hline & $\mathrm{M}_{\text {mean }}=4.6$ & $M_{\text {mean }}=12.66$ \\
\hline & $\mathrm{M}_{\max }=6.72$ & $\mathrm{M}_{\max }=18.3$ \\
\hline \multirow{3}{*}{ JPEG QF $=50 \%$} & $\mathrm{M}_{\min }=3.7$ & $\mathrm{M}_{\min }=10.2$ \\
\hline & $\mathrm{M}_{\text {mean }}=4.74$ & $\mathrm{M}_{\text {mean }}=12.63$ \\
\hline & $\mathrm{M}_{\max }=6.17$ & $\mathrm{M}_{\max }=18$ \\
\hline \multirow{3}{*}{ JPEG QF $=30 \%$} & $\mathrm{M}_{\min }=3.12$ & $\mathrm{M}_{\min }=8.51$ \\
\hline & $\mathrm{M}_{\text {mean }}=4.3$ & $\mathrm{M}_{\text {mean }}=10.9$ \\
\hline & $\mathrm{M}_{\max }=5.77$ & $\mathrm{M}_{\max }=16.45$ \\
\hline \multirow{3}{*}{$\begin{array}{l}\text { JPEG2000 } \\
\text { quantization } p=5\end{array}$} & $\mathrm{M}_{\min }=3.66$ & $\mathrm{M}_{\min }=7.11$ \\
\hline & $\mathrm{M}_{\text {mean }}=4.5$ & $\mathrm{M}_{\text {mean }}=10$ \\
\hline & $\mathrm{M}_{\max }=5.6$ & $\mathrm{M}_{\max }=13.35$ \\
\hline
\end{tabular}


Table 4 Maximal, minimal and mean detection measures $M$ for a set of tested images under geometrical attacks

\begin{tabular}{|c|c|c|}
\hline $\begin{array}{c}\text { Test } \\
\text { Statistics }\end{array}$ & $\begin{array}{l}\text { Standard } \\
\text { correlator }\end{array}$ & $\begin{array}{l}\text { Optimal } \\
\text { detector }\end{array}$ \\
\hline \multirow{3}{*}{$\begin{array}{l}\text { Cropping } \\
3 \text { rows and } 3 \\
\text { cols. }\end{array}$} & $\mathrm{M}_{\min }=3.4$ & $\mathrm{M}_{\min }=5.4$ \\
\hline & $M_{\text {mean }}=4.6$ & $\mathrm{M}_{\text {mean }}=12$ \\
\hline & $\mathrm{M}_{\max }=5.66$ & $M_{\max }=19.7$ \\
\hline \multirow{3}{*}{ Rotation $1^{\circ}$} & $\mathrm{M}_{\min }=3.33$ & $\mathrm{M}_{\min }=5.11$ \\
\hline & $\mathrm{M}_{\text {mean }}=5.5$ & $M_{\text {mean }}=9.6$ \\
\hline & $\mathrm{M}_{\max }=13.56$ & $\mathrm{M}_{\max }=10.5$ \\
\hline \multirow{3}{*}{ Rescale $90 \%$} & $\mathrm{M}_{\min }=3.26$ & $\mathrm{M}_{\min }=3.9$ \\
\hline & $\mathrm{M}_{\text {mean }}=4.35$ & $\mathrm{M}_{\text {mean }}=7.34$ \\
\hline & $\mathrm{M}_{\max }=5$ & $\mathrm{M}_{\max }=9.65$ \\
\hline
\end{tabular}

\subsubsection{Comparison results}

The results of the proposed procedure are compared with the results of two procedures in the DCT domain and DWT approaches based on the GGF and Cauchy pdf model. The measures M of detection quality under attacks are given in Table 5. Some details for these procedures are given in the sequel.

1) The procedure proposed in [10], can be considered as DCT counterpart of the proposed DWT procedure. Instead of JPEG2000 algorithm, it is based on the standard JPEG. The specific DCT coefficients from the $8 \times 8$ blocks are selected for watermarking. The detection is performed by using the optimal detector (similarly as in the case of proposed procedure). The results are given in the Table 5 ( $2^{\text {nd }}$ column).

2) The second approach is commonly used standard DCT procedure in the 8x8 DCT domain: all middle frequency coefficients from 8x8 DCT blocks are used for watermarking (22000 coefficients for images of size $256 \times 256$ ). A standard additive watermark embedding procedure is used. The detection is performed by using the standard correlation detector (Table $5,3^{\text {rd }}$ column).

3) Finally, we consider the standard DWT domain watermarking. The watermark detection in the DWT subbands is usually based on the Generalized Gaussian or Cauchy pdf [4], [5]. Namely, if the watermarking coefficients are chosen without the selection criteria proposed in this paper, then their pdf may correspond either to GGF or Cauchy function, and detection is performed by using one of the mentioned pdf models [5]. In this case, we need a higher number of coefficients for 
reliable detection results, and thus 4000 DWT coefficients has been used (twice higher than in the proposed procedure). Further increasing of the coefficients number will not influence significantly the detection index. The results are given in Table $5\left(4^{\text {th }}\right.$ and $5^{\text {th }}$ column) for the GGF and Cauchy pdf model (the values of parameters are chosen experimentally such that to provide the highest detector response).

The watermark is embedded with the same PSNR $\approx 47 \mathrm{~dB}$ in all cases. Note that the proposed approach provides more reliable results the other two considered procedures.

Table 5. Comparison with other procedures

\begin{tabular}{|c|c|c|c|c|c|}
\hline $\begin{array}{c}\text { Measure of detection } \\
\text { quality (M) }\end{array}$ & $\begin{array}{c}\text { Optimal } \\
\text { detection in } \\
\text { DWT } \\
\text { (proposed) }\end{array}$ & $\begin{array}{c}\text { Optimal } \\
\text { detection } \\
\text { in DCT } \\
\text { (1) }\end{array}$ & $\begin{array}{c}\text { Standard } \\
\text { DCT } \\
\text { Procedure } \\
\text { (2) }\end{array}$ & $\begin{array}{c}\text { DWT } \\
\text { watermarking } \\
\text { GGF detector } \\
\text { (3) }\end{array}$ & $\begin{array}{c}\text { DWT } \\
\text { watermarking } \\
\text { Cauchy } \\
\text { detector } \\
\text { (3) }\end{array}$ \\
\hline No attack & 13.5 & 12.6 & 8.46 & 5.2 & 5.1 \\
\hline Gaussian noise & 7.5 & 3.9 & 5.4 & 4.7 & 3.8 \\
\hline Impulse noise & 12 & 6.8 & 7.8 & 5 & 4.7 \\
\hline Median filter & 12.7 & 5.3 & 4.1 & 4.9 & 4.3 \\
\hline JPEG QF $=50 \%$ & 12.6 & 10.9 & 5.1 & 4.1 & 4.5 \\
\hline JPEG QF $=30 \%$ & 10.9 & N/A & 4.13 & 4 & 4.4 \\
\hline
\end{tabular}

\subsubsection{Capacity}

As described in the previous Section, the watermark is created as a pseudo-noise sequence. The number of watermarked coefficients (belonging to the III subband) is between 1200 and 2000 for different test images. It has been shown that the minimal number of coefficients required for reliable detection is approximately 100 (with Perr $\approx 10^{-4}$ ). Thus, the watermark bit can be represented as a pseudo-noise sequence having 100 random elements. According to this scenario, the number of embedded bits is between 12 and 20. However, if required, the number of embedded bits can be increased: a) by using lower value of $C$, eg. $C=30$, which would provide higher number of coefficients (close to 3000 coefficients), b) by including the coefficients from other subbands. 


\subsection{Example2}

In this example, the proposed procedure is tested for different numbers of decomposition levels (from 3 to 7 levels). The watermark has been embedded in different subbands (II, III, IV, V, VI) to test which subband is the most appropriate for watermarking. The tests are performed with the same value of watermark embedding strength $\alpha=0.1$. The measures of detection quality together with image quality metrics (VSNR, PSNR and CSNR) are reported in Table 6 and Table 7.

Table 6. Detection measures and image quality metrics

\begin{tabular}{|c|c|c|c|c|c|c|}
\hline \multirow{2}{*}{\multicolumn{2}{|c|}{$\begin{array}{c}\mathrm{C}=40 \\
\text { No attacks }\end{array}$}} & \multicolumn{5}{|c|}{ NUMBER OF DECOMPOSITION LEVELS } \\
\hline & & 3 & 4 & 5 & 6 & 7 \\
\hline \multirow{6}{*}{ 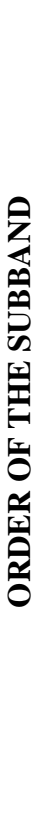 } & I & & & & & \\
\hline & II & $\begin{array}{c}\mathbf{M}=\mathbf{1 5 . 1 9 6 9} \\
\alpha=0.1 ; \\
\text { PSNR }=51.12 \\
\text { VSNR }=42.00 \\
\text { CPSNR }=51.84\end{array}$ & $\begin{array}{c}\mathbf{M}=\mathbf{9 . 1 6 0 0} \\
\alpha=0.1 ; \\
\text { PSNR }=53.43 \\
\text { VSNR }=43.39 \\
\text { CPSNR }=54.17\end{array}$ & $\begin{array}{c}\mathbf{M}=\mathbf{3 . 3 6 2 3} \\
\alpha=0.1 ; \\
\text { PSNR }=62.80 \\
\text { VSNR }=\text { Inf } \\
\text { CPSNR }=63.46\end{array}$ & $\begin{array}{c}\mathbf{M}=\mathbf{0 . 2 4 6 3} \\
\alpha=0.1 ; \\
\text { PSNR=63.44 } \\
\text { VSNR=Inf } \\
\text { CPSNR=63.89 }\end{array}$ & \\
\hline & III & $\begin{array}{c}\mathbf{M}=\mathbf{1 2 . 9 2 6 5} \\
\alpha=0.1 ; \\
\text { PSNR }=46.12 \\
\text { VSNR }=36.38 \\
\text { CPSNR }=46.83\end{array}$ & $\begin{array}{c}\mathbf{M}=\mathbf{1 2 . 1 7 2 2} \\
\alpha=0.1 ; \\
\text { PSNR }=46.57 \\
\text { VSNR }=37.10 \\
\text { CPSNR }=47.33\end{array}$ & $\begin{array}{c}\mathbf{M}=\mathbf{1 2 . 2 6 1 5} \\
\alpha=0.1 ; \\
\text { PSNR }=47.56 \\
\text { VSNR }=38.70 \\
\text { CPSNR }=48.21\end{array}$ & $\begin{array}{c}\mathbf{M}=\mathbf{8 . 4 8 9 1} \\
\alpha=0.1 ; \\
\text { PSNR }=48.48 \\
\text { VSNR }=39.12 \\
\text { CPSNR }=49.01\end{array}$ & $\begin{array}{c}\mathbf{M}=\mathbf{5 . 2 6 9 2} \\
\alpha=0.1 ; \\
\text { PSNR }=54.42 \\
\text { VSNR }=44.16 \\
\text { CPSNR }=54.78\end{array}$ \\
\hline & IV & $X$ & $\begin{array}{c}\text { M=7.0898 } \\
\alpha=0.1 ; \\
\text { PSNR }=43.99 \\
\text { VSNR }=33.65 \\
\text { CPSNR }=44.75\end{array}$ & $\begin{array}{c}\mathbf{M}=\mathbf{6 . 6 5 3 2} \\
\alpha=0.1 ; \\
\text { PSNR }=44.31 \\
\text { VSNR }=33.92 \\
\text { CPSNR }=44.87\end{array}$ & $\begin{array}{c}\mathbf{M}=\mathbf{7 . 6 0 6 2} \\
\alpha=0.1 ; \\
\text { PSNR }=43.74 \\
\text { VSNR=33.19 } \\
\text { CPSNR }=44.27\end{array}$ & $\begin{array}{c}\text { M=7.8092 } \\
\alpha=0.1 ; \\
\text { PSNR }=44.91 \\
\text { VSNR }=34.67 \\
\text { CPSNR }=45.28\end{array}$ \\
\hline & $\mathbf{V}$ & $X$ & $X$ & $\begin{array}{c}\mathbf{M}=\mathbf{2 . 3 5 2 3} \\
\alpha=0.1 ; \\
\text { PSNR }=40.44 \\
\text { VSNR }=29.98 \\
\text { CPSNR }=41.10\end{array}$ & $\begin{array}{c}\mathbf{M}=\mathbf{2 . 2 6 4 5} \\
\alpha=0.1 ; \\
\text { PSNR=39.92 } \\
\text { VSNR=29.14 } \\
\text { CPSNR }=40.45\end{array}$ & $\begin{array}{c}\mathbf{M}=\mathbf{3 . 2 8 9 2} \\
\alpha=0.1 ; \\
\text { PSNR }=41.85 \\
\text { VSNR }=31.29 \\
\text { CPSNR }=42.24\end{array}$ \\
\hline & VI & $X$ & $X$ & $X$ & $\begin{array}{c}\mathbf{M}=\mathbf{2 . 6 5 1 1} \\
\alpha=0.1 ; \\
\text { PSNR }=38.04 \\
\text { VSNR }=28.78 \\
\text { CPSNR }=38.58\end{array}$ & $\begin{array}{c}\mathbf{M}=\mathbf{2 . 2 1 8 9} \\
\alpha=0.1 ; \\
\text { PSNR }=38.19 \\
\text { VSNR }=28.83 \\
\text { CPSNR }=38.58\end{array}$ \\
\hline
\end{tabular}


Table 7. Detection measures and image quality metrics

\begin{tabular}{|c|c|c|c|c|c|c|}
\hline \multirow{2}{*}{\multicolumn{2}{|c|}{$\begin{array}{c}\mathrm{C}=40 \\
\text { Gaussian } \\
\text { noise }\end{array}$}} & \multicolumn{5}{|c|}{ NUMBER OF DECOMPOSITION LEVELS } \\
\hline & & \multirow[t]{2}{*}{3} & \multirow[t]{2}{*}{4} & \multirow[t]{2}{*}{5} & \multirow[t]{2}{*}{6} & \multirow[t]{2}{*}{7} \\
\hline \multirow{6}{*}{ 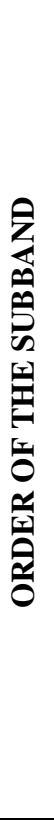 } & $\mathbf{I}$ & & & & & \\
\hline & II & $\begin{array}{l}\mathbf{M}=\mathbf{1 . 9 3 5 0} \\
\alpha=0.1 ; \\
\text { PSNR }=51.12 \\
\text { VSNR }=42.00 \\
\text { CPSNR }=51.84 \\
\end{array}$ & \begin{tabular}{|l|}
$M=5.3966$ \\
$\alpha=0.1 ;$ \\
PSNR $=53.43$ \\
VSNR=43.39 \\
CPSNR $=54.17$ \\
\end{tabular} & \begin{tabular}{|l|}
$\mathbf{M}=\mathbf{1 . 5 6 8 6}$ \\
$\alpha=0.1 ;$ \\
PSNR $=62.80$ \\
VSNR=Inf \\
CPSNR $=63.46$ \\
\end{tabular} & $\begin{array}{l}\mathbf{M}=\mathbf{0 . 4 7 2 0} \\
\alpha=0.1 ; \\
\text { PSNR }=63.44 \\
\text { VSNR=Inf } \\
\text { CPSNR }=63.89 \\
\end{array}$ & \\
\hline & III & $\begin{array}{c}\mathbf{M}=\mathbf{0 . 7 8 4 8} \\
\alpha=0.1 ; \\
\text { PSNR }=46.12 \\
\text { VSNR=36.38 } \\
\text { CPSNR }=46.83 \\
\end{array}$ & \begin{tabular}{|c|}
$\mathbf{M}=\mathbf{1 . 8 3 2 9}$ \\
$\alpha=0.1 ;$ \\
PSNR $=46.57$ \\
VSNR=37.10 \\
CPSNR $=47.33$ \\
\end{tabular} & \begin{tabular}{|c|}
$\mathbf{M}=\mathbf{7 . 4 7 2 5}$ \\
$\alpha=0.1 ;$ \\
$\mathrm{PSNR}=47.56$ \\
VSNR $=38.70$ \\
CPSNR $=48.21$
\end{tabular} & $\begin{array}{l}\mathbf{M}=\mathbf{6 . 0 1 3 6} \\
\alpha=0.1 ; \\
\text { PSNR }=48.48 \\
\text { VSNR }=39.12 \\
\text { CPSNR }=49.01\end{array}$ & $\begin{array}{c}\mathbf{M}=\mathbf{4 . 0 7 5 9} \\
\alpha=0.1 ; \\
\text { PSNR }=54.42 \\
\text { VSNR=44.16 } \\
\text { CPSNR }=54.78\end{array}$ \\
\hline & IV & $\mathrm{X}$ & \begin{tabular}{|l|}
$\mathbf{M}=\mathbf{0 . 5 0 8 9}$ \\
$\alpha=0.1 ;$ \\
PSNR $=43.99$ \\
VSNR $=33.65$ \\
CPSNR $=44.75$ \\
\end{tabular} & $\begin{array}{l}\mathbf{M}=\mathbf{1 . 1 3 6 5} \\
\alpha=0.1 ; \\
\text { PSNR }=44.31 \\
\text { VSNR }=33.92 \\
\text { CPSNR }=44.87\end{array}$ & $\begin{array}{l}\mathbf{M}=\mathbf{6 . 5 7 1 0} \\
\alpha=0.1 ; \\
\text { PSNR=43.74 } \\
\text { VSNR=33.19 } \\
\text { CPSNR }=44.27\end{array}$ & $\begin{array}{l}\mathbf{M}=\mathbf{6 . 8 1 0 1} \\
\alpha=0.1 ; \\
\text { PSNR }=44.91 \\
\text { VSNR }=34.67 \\
\text { CPSNR }=45.28 \\
\end{array}$ \\
\hline & $\mathbf{V}$ & $X$ & $X$ & \begin{tabular}{|l}
$\mathbf{M}=\mathbf{0 . 7 7 4 9}$ \\
$\alpha=0.1 ;$ \\
PSNR $=40.44$ \\
VSNR $=29.98$ \\
CPSNR $=41.10$
\end{tabular} & $\begin{array}{l}\mathbf{M}=\mathbf{1 . 4 2 2 4} \\
\alpha=0.1 ; \\
\text { PSNR=39.92 } \\
\text { VSNR=29.14 } \\
\text { CPSNR }=40.45\end{array}$ & $\begin{array}{l}\mathbf{M}=\mathbf{2 . 5 9 0 3} \\
\alpha=0.1 ; \\
\text { PSNR }=41.85 \\
\text { VSNR }=31.29 \\
\text { CPSNR }=42.24\end{array}$ \\
\hline & VI & $\mathrm{X}$ & $\mathrm{X}$ & $\mathrm{X}$ & \begin{tabular}{l}
\multicolumn{1}{c}{$\mathbf{M}=\mathbf{1 . 2 6 7 1}$} \\
$\alpha=0.1 ;$ \\
PSNR $=38.04$ \\
VSNR=28.78 \\
CPSNR $=38.58$ \\
\end{tabular} & $\begin{array}{c}\mathbf{M}=\mathbf{1 . 6 5 5 2} \\
\alpha=0.1 ; \\
\text { PSNR }=38.19 \\
\text { VSNR }=28.83 \\
\text { CPSNR }=38.58\end{array}$ \\
\hline
\end{tabular}

We present the results in the case of no attacks (Table 6) and in the presence of Gaussian noise (Table 7), which affects optimal detection more than other considered attacks. It has been shown that watermark embedding in the III subband of the 5 level decomposition scheme (which can be denoted as 5-III), provides in the same time high value of $M$ and high values of image quality metrics (PSNR, VSNR and CPSNR) even under attacks. The other candidates such as 3-II, 3-III, 4-III from Table 6, do not provide reliable detection under attacks (Table 7).

Simulation results summary: Finally, we can summarize the results obtained by the analysis and simulation of the proposed procedure as follows. The watermark embedding should be done by using the coefficients belonging to the III subband of the 5 level decomposition scheme. In this way the highest value of detection measure $M$ and highest values of image quality metrics (PSNR, VSNR and CPSNR) is provided. The JPEG2000 quantization is applied to the coefficients in the III subband, with the step $q$ calculated by using (3) (the parameter $p=8$ is used). The watermarking coefficients are selected according to: $\left|I_{q}(x, y)\right|>C \cdot q$, where the optimal value of gap parameter $C\left(C_{o p t}=40\right)$ is chosen to provide the robustness for all considered attacks (impulse and Gaussian 
noise, filtering, JPEG2000, JPEG, rotation, scaling and cropping). It has been shown that the scaling factor used to control the watermark strength should be $\alpha=0.1$, providing the PSNR $\approx 4 \mathrm{~dB}$.

\section{CONCLUSION}

The watermarking procedure based on the biorthogonal irreversible $(9,7)$ floating point wavelets is proposed. The suitable choice of wavelet subbands and their coefficients leads to the good tradeoff between the watermark imperceptibility and robustness. Efficient detection results are obtained by using an optimal detector form derived from the coefficients pdf. The procedure has been tested in the presence of various attacks and it provides high measures of detection quality. In the future work, an analytical expression for optimal gap parameter selection can be derived. The future work may also include the application of the proposed approach in detection of illegal use of multimedia content, in order to enhance the security for multimedia forensics mechanisms [32]. In that sense, it would be interesting to consider the combination of the extrinsic content protection (watermarking) with the intrinsic fingerprint methods [33], as well as the interaction between multimedia forensics and network adaptation [34].

\section{ACKNOWLEDGEMENT}

The authors are thankful to the reviewers for useful comments and suggestions that helped to improve the quality of the paper.

\section{REFERENCES}

[1] Cox. I., Miller M. and Bloom, J., 'Digital watermarking: principles and practice', Morgan Kaufmann, (2002)

[2] Katzenbeisser, S., Petitcolas, F., 'Information Hiding: Techniques for steganography and digital watermarking', Artech House, (2000)

[3] Stanković, S., Djurović, I. and Pitas, I., 'Watermarking in the space/spatial-frequency domain using two-dimensional Radon Wigner distribution' IEEE Trans. on Image Process., vol. 10, No. 4, pp. 650 - 658, (2001) 
[4] Nikolaidis, A., and Pitas, I., 'Asymptotically optimal detection for additive watermarking in the DCT and DWT domains', IEEE Trans. on Image Process., vol. 12 No.5: 563-571, (2003)

[5] R. Kwitt, P. Meerwald, A. Uhl, 'Efficient Detection of Additive DWT Watermarking in the DWT domain' 17th European Signal Processing Conference, EUSIPCO 2009, Aug. 2009, pp. 2072-2076.

[6] Cheng, Q. and Huang, T., 'Robust optimum detection of transform domain multiplicative watermarks', IEEE Trans. Signal Process., vol. 51, No. 4, pp. 906-924, (2003)

[7] Hernandez, J. R., Amado, M., and Perez Gonzales, F., 'DCT domain watermarking techniques for still image: Detector performance analysis and a new structure', IEEE Trans. Image Process., vol. 9, pp. 55 -68, (2000)

[8] Stanković, S., Djurović, I., Herpers, R., and Stanković, LJ., 'An approach to the optimal watermark detection', AEU, Int. J. Electron. Commun., vol. 57, pp.355 - 357, (2003)

[9] Briassouli, A., and Strintzis, M. G., 'Locally Optimum Nonlinearities for DCT Watermark Detection', IEEE Transaction on Image Processing, vol. 13, No. 12, pp. 1604-1618, Dec. 2004.

[10] Briassouli, A., Tsakalides, P., and Stouraitis, A., 'Hidden messages in heavy-tails: DCTdomain watermark detection using alpha stable models' IEEE Trans. on Multimedia 7(4), 700-715, 2005.

[11] Stanković, S., Orović, I., Žarić, N., 'Robust watermarking procedure based on JPEG direct cosine transform image compression', Journal of Electronic Imaging, vol. 17, No. 4, pp. 043001, (2008)

[12] Vahedi, E., Zoroofi, R. A., and Shiva, M., 'On Optimal Parameter Selection for Multiresolution Based Image Watermarking', Int. Conf. on Intelligent and Advanced Systems 2007, ICIAS 2007, pp.641-646, Nov. 2007.

[13] Vahedi, E., Zoroofi, R. A., and Shiva, M., 'On Optimal Color Coordinate Selection for Wavelet-Based Color Image Watermarking'. Int. Conf. on Intelligent and Advanced Systems 2007, ICIAS 2007, pp.635-640, Nov. 2007.

[14] Meerwald, P., and Uhl, A., 'A Survey of Wavelet-domain Watermarking Algorithm', in Proceedings of SPIE, Electronic Imaging, Security and Watermarking of Multimedia Contents III, vol. 4314, CA, USA, Jan. 2001, pp. 501-516.

[15] Agreste, S., Andaloro, G., Prestipino, D., and Puccio, L., 'An Image Adaptive, Waveletbased Watermarking of digital images', Journal of Computational and Applied Mathematics, Vol. 210, Issue 1-2, December, 2007.

[16] Mehul, R., and Priti, R., 'Discrete Wavelet Transform Based Multiple Watermarking Scheme', Proceedings of IEEE Region 10 Technical Conference on Convergent Technologies for the Asia-Pacific, India, 2003.

[17] Y.-S. Seo, M.-S. Kim,H.-J. Park, H.-Y. Jung and H.-Youl. Chung, "A Secure Watrmarking for JPEG-2000," International Conference on Image Processing, ICIP 2001, vol. 2, pp.530-533. 
[18] G.C. Langelaar, I. Setyawn, R.L. Lagendijk, "Watermarking digital image and video data, A state-of-the-art overview," IEEE Signal Processing Magazine, vol. 17, no.5, pp. 20-46, 2000.

[19] S. Joo, Y. Suh, J. Shin, and H. Kikuchi, “A New Robust Watermarking Embedding into Wavelet DC Components”, ETRI Journal, Volume 24, No. 5, 2002.

[20] K. Veeraswamy, S. SrinivasKumar, "An Improved Wavelet based Image Compression Scheme and Oblivious Watermarking," IJCSNS International Journal of Computer Science and Network Security, vol. 8, no. 4, April 2008.

[21] Y.-H. Seo, H.-J. Choi, J.-S. Yoo, and D.-W. Kim, “Adaptive Watermarking Scheme Using Biased Shift of Quantization Index," Journal of Electrical and Computer Engineering, Volume 2010 (2010), Article ID 348321, 6 pages, doi:10.1155/2010/348321

[22] Orlandić, M., Orović, I., Stanković, S., 'An Image Watermarking Technique with Optimal Detection in the Wavelet Transform Domain', 53rd ETRAN Conference, Vrnjačka Banja, Serbia, 2009.

[23] Daubechies, I., 'Ten Lectures on Wavelets', Regional Conference Series in Applied Mathematics, SIAM, Philadelphia, (1992).

[24] Daubechies I., and Sweldens, W., 'Factoring Wavelet Transforms into Lifting Steps', J. Fourier Anal. Appl., vol. 4, No. 3, pp. 245-267, (1998)

[25] Dia, D., Zeghid, M., Saidani, T., Atri, M., Bouallegue, B., Machhout, M., and Tourki, R., 'Multi-level Discrete Wavelet Transform Architecture Design', Proc. of the World Congress on Engineering 2009, vol. 1, WCE 2009, July 1 - 3, 2009.

[26] Marcellin, M. W., Lepley, MA. A., Bilgin, A., Flohr, T. J., Chinen, T. T., and Kasner, J. H., 'An overview of quantization in JPEG2000', Signal Processing: Image Communication, Elsevier, 17(1):73-84, 2002.

[27] Chandler, D.M., Hemami, S.S., 'VSNR: A Wavelet-Based Visual Signal-to-Noise Ratio for Natural Images', IEEE Transactions on Image Processing, vol. 16, No. 9, pp. 2284 2298.

[28] Mukherjee, J., Lang, M.K., and Mitra, S.K., 'False Color Suppression in Demosaiced Color Images', ICVGIP-2004, Kolkata, Dec. 2004, pp. 320-325.

[29] Tay, P.C., Havlicek, J.P., 'JPEG 2000 scalar quantization using an optimally frequency localized modulated lapped transform', IEEE Int. Conf on Image Processing 2005, ICIP 2005, pp. I-93-6.

[30] Brown, C. L., and Zoubir, A. M., 'A nonparametric approach to signal detection in impulsive interference', IEEE Transaction on Signal Processing, vol. 42, pp. 2665-2669, 2000.

[31] Wickens, T. D., 'Elementary Signal Detection Theory', Oxford, U.K.: Oxford Univ. Press, 2002.

[32] Battiato, Emmanuel, S., Ulges, A., Worring, M., 'Multimedia in Forensics, Security, and Intelligence', IEEE Multimedia Magazine, vol. 19, Issue 1, pp. 17-19, 2012. 
[33] Lin, W.S., Tjoa, S.K., Zhao, H.V., Liu, K.J.R., 'Digital image source coder forensics via intrinsic fingerprints' IEEE Transactions on Information Forensics and Security, vol. 4, No. 3, pp. 460-475, 2009.

[34] Zhou, L., Chao, H._C., Vasilakos, A.V., 'Joint Forensics-Scheduling Strategy for DelaySensitive Multimedia Applications over Heterogeneous Networks' IEEE Journal on Selected Areas in Communications, Vol. 29, No. 7, pp.1358-1367, 2011. 